\title{
Evaluation of the MGIT 960/EpiCenter TB eXiST system for drug susceptibility testing for Mycobacterium abscessus complex
}

Natalia de CARVALHO

Instituto Adolpho Lutz

Sylvia LEÃO

Universidade Federal de Sao Paulo - Campus Diadema

\section{Sidney Bombarda}

Divisao de Tubercolose - Centro de Vigilancia Epidemiologica do Estado de Sao Paulo (CVE)

\section{Robert D Arbeit}

Tufts Medical Center https://orcid.org/0000-0002-7013-3269

Erica CHIMARA ( $\nabla$ erica.chimara@ial.sp.gov.br)

Instituto Adolfo Lutz

Research article

Keywords: Mycobacterium abscessus complex, Drug susceptibility testing, REMA, BACTEC MGIT 960, TB eXiST system

Posted Date: January 21st, 2021

DOl: https://doi.org/10.21203/rs.3.rs-62195/v2

License: (9) (1) This work is licensed under a Creative Commons Attribution 4.0 International License. Read Full License 


\section{Abstract}

Background: Drug susceptibility testing (DST) of the Mycobacterium abscessus complex (MABSC) and other rapidly growing nontuberculous mycobacteria by conventional broth microdilution (BMD) is complicated due to inducible resistance to clarithromycin and other technical factors. The goals of this study were to develop a protocol for performing DST of MABSC clinical isolates on the BACTEC MGIT 960/Epicenter TB eXiST and provide an initial assessment of its reliability and utility.

Methods: M. abscessus ATCC19977 was used as the reference strain for developing the protocol and as the internal control for DST done by BMD method per CLSI guidelines plus resazurin. Both methods were applied to 31 MABSC clinical isolates submitted to our reference center. Genotyping was performed for the $r r l$ and erm(41) genes known to impact clarithromycin resistance phenotype.

Results: The 31 MABSC isolates included 14 M. abscessus subsp. abscessus, 8 M. abscessus subsp. massiliense, and 9 M. abscessus subsp. bolletii. By standard BMD method, a high percentage of the isolates were resistant to cefoxitin (93.5\%) and imipenem (100\%), and sensitive to amikacin (96.7\%). Comparing microplate and MGIT 960 results across those 93 pairs of results (31 isolates $\times 3$ antibiotics), $75(80.6 \%)$ were concordant and the remaining 18 (19.4\%) represented minor errors; there were no major or very major errors. Concordance was $100 \%$ for amikacin, $84 \%$ for imipenem, and $58 \%$ for cefoxitin. Clarithromycin DST by microplate and MGIT 960 both identified $14(45.2 \%)$ isolates as susceptible. Microplate identified 3 (9.6\%) isolates as resistant after 3 days incubation, with 14 (45.2\%) demonstrating inducible resistance from Day 5 to 14. Among those 17 isolates, the MGIT 960 protocol, without modifications, reported $9(29.0 \%)$ as resistant and 8 (25.8\%) as intermediate. For all isolates, the observed clarithromycin susceptibility phenotypes were consistent with the genotypes.

Conclusion: This study details the development of a DST protocol for MABSC isolates using the MGIT 960/Epicenter TB eXiST system. Based on direct comparison with the standard BMD, the protocol provided highly reliable results, including, without further modification, detection of isolates with inducible-resistance to clarithromycin. These findings support proceeding to the multi-laboratory collaborative study required for validation of the protocol.

\section{Introduction}

Isolates of the Mycobacterium abscessus complex (MABSC) represent the most frequent species of rapidly growing mycobacteria (RGM) causing clinically significant infection, accounting for $80 \%$ of lung disease caused by $\mathrm{RGM}^{1-4}$. MABSC isolates are widely distributed in the environment and have been associated with both nosocomial and community-acquired opportunistic infections in compromised hosts and in persons with underlying chronic lung disease, including patients with cystic fibrosis (CF $)^{1-4}$. With advances in diagnostic methods, there has been a worldwide increase in the reported incidence of MABSC infections ${ }^{5}$. 
Treatment of these infections is substantially complicated by the high frequency of resistance to many antimicrobials. Consequently, effective management requires drug susceptibility testing (DST) of each clinical isolate, which in turn is complicated by the prevalence of the clarithromycin inducible resistance phenotype p.6. $^{5,6}$.

The DST technique recommended by the Clinical and Laboratory Standards Institute (CLSI) is the Broth Microdilution (BMD) method for determining the minimal inhibitory concentration (MIC), which is defined as the lowest concentration of the drug that inhibits the growth of the microorganism. Although this approach is well-established, there are technical challenges in applying the method to RGM.

Resazurin is an oxidation-reduction indicator useful for demonstrating bacterial growth. The resazurin microtiter assay (REMA) modification to the BMD method of DST makes visual readings of the microwell cultures easier and facilitates consistency among different observers. This approach has been widely applied to DST of $M$. tuberculosis (MTB) and endorsed by WHO7,8,9. Carvalho et al. (2016) ${ }^{10}$ reported a standardized BMD method incorporating resazurin for DST of MABSC isolates, with particular attention to detection of induced-resistance to clarithromycin (CLR) with extended incubation. The benefits of this modification have been confirmed by other laboratories ${ }^{11,12,13}$.

The fully-automated BACTEC Mycobacterium Growth Indicator Tube 960 system (MGIT 960), as initially released by Becton-Dickinson (Franklin Lakes, NJ, USA) with Epicenter software, focused on drug susceptibility testing for MTB using prepared tubes provided by the manufacturer. Based on World Health Organization (WHO) recommendations that liquid media are preferred for both culture ${ }^{14}$ and susceptibility testing ${ }^{15}$ of $M$. tuberculosis (MTB), the MGIT 960 system became established in many reference laboratories charged with providing those services, including the network of public and private laboratories in the state of São Paulo, Brazil ${ }^{10}$. The subsequent addition of the TB eXist module permitted the implementation of additional, user-defined protocols, thereby enabling the system to be applied to additional drugs and concentrations as well as non-tuberculous mycobacteria. Recent publications have validated protocols for susceptibility testing of slow-growing nontuberculous mycobacteria ${ }^{16,17,18}$.

This report describes the development of a method for applying the BACTEC MGIT 960 system with TB eXiST software to DST of rapidly-growing mycobacteria. As expected, a different protocol was required, which was then applied to a set of 31 clinical isolates of MABSC. The categorical results obtained using the BACTEC MGIT 960 system are compared with those obtained by REMA.

\section{Material And Methods}

\subsection{Bacterial strains}

We selected 31 clinical isolates of MABSC submitted by outside clinical laboratories for species confirmation and DST to the Tuberculosis and Mycobacteriosis Laboratory at the Adolfo Lutz Institute, São Paulo, Brazil. The isolates were diverse as regards geography (22 different laboratories in 15 
different cities), time of collection (from January 2010 to December 2011), and source (13 sputum, 5 bronchoalveolar lavage, and 13 unspecified sites, representing 28 different patients). Subspecies identification was performed by PRA- $h s p 65$ (PCR-restriction enzyme analysis of the $h s p 65$ gene) and confirmed by rpoB gene sequencing ${ }^{19,20}$. M. abscessus ATCC $19977^{\top}$ reference strain was used for assay development and as the control strain during subsequent studies.

\subsection{Genotyping clarithromycin resistance loci}

Mutations in erm(41) and $r r l$ genes associated with resistance and susceptibility to CLR were identified using the protocol of Carvalho et al. (2018) ${ }^{21}$. The sequences were analyzed using BioNumerics version 7.1 (Applied Maths, TX, USA). The reference sequence for the erm(41) gene was that of $M$. abscessus MAB 30 (Genbank number EU590129), and for $r r l$ gene, M. abscessus ATCC $19977^{\top}$ (Genbank number NC010397.1).

\subsection{Minimal Inhibitory Concentration Determination by microplate method}

The MIC protocol was performed as recommended by CLSI $(2018)^{6}$, using 96-well microplates and cation-adjusted Mueller-Hinton broth (CAMHB) without Oleic Albumin Dextrose Catalase (OADC) growth supplement, modified by inclusion of the vital stain resazurin as color indicator to facilitate visual growth detection (Resazurin Microtiter Assay [REMA])(Carvalho et al. 2016) ${ }^{10}$. Because the standard temperature for the MGIT 960 is $35 \pm 2^{\circ} \mathrm{C}$, the microplates were also incubated at that temperature, rather than usual $30 \pm 2^{\circ} \mathrm{C}$. We had previously done DST at the usual temperature for all the isolates used and confirmed the microplate susceptibility results were the same at both temperatures.

The drugs tested were CLR, amikacin (AMK), cefoxitin (FOX) and imipenem (IPM), which represent agents recommended for the treatment of $M$. abscessus infections by the American Thoracic Society (ATS $)^{16}$. Two types of plates were prepared, one with only $\operatorname{CLR}(0,5-64 \mathrm{mg} / \mathrm{L})$ and the other with the three remaining antibiotics tested (AMK $4-512 \mathrm{mg} / \mathrm{L}$; FOX 4 - $512 \mathrm{mg} / \mathrm{L}$; IMP $1-128 \mathrm{mg} / \mathrm{L}$ ). This design was chosen to permit assessment of clarithromycin-induced resistance, which requires an extended incubation period of 14 days ${ }^{17}$. The MIC was defined as the lowest drug concentration that prevented growth; susceptible, intermediate, and resistant were defined using the breakpoint concentrations recommended by CLSI (2018). For CLR, isolates that met criteria for susceptibility on Day 3, but were classified as resistant at one of the subsequent readings on Days $5,7,10$, and 14 , were reported as "inducible resistance" (IR).

\subsection{Inoculum standardization for drug susceptibility testing using the MGIT 960}

The manufacturer recommendations for applying the BACTEC MGIT 960 with TB eXiST V3.00D For EpiCenter $^{\text {TM }}$ V6.20A for DST specify (a) a media only growth control using a standard inoculum, determined empirically, which generates a growth signal of 400 growth units (GU) after at least 72 hours (3.0 days) incubation and (b) using a 100-fold higher inoculum of organisms for the tubes with varying drug concentrations. 
To determine the appropriate inocula and media for MABSC, a bacterial suspension of 0.5 McFarland of the ATCC $19977^{\top}$ was prepared in sterile distilled water and used to prepare dilutions ranging from $10^{-1}$ to $10^{-8}$. The tubes provided for the MGIT 960 system contain $7.0 \mathrm{~mL}$ of $7 \mathrm{H} 9$ media, were prepared with or without $0.8 \mathrm{~mL}$ of OADC supplementation, and then inoculated with $0.5 \mathrm{~mL}$ aliquots of the bacterial dilutions.

\subsection{Drug susceptibility testing using the BACTEC MGIT 960 with TB eXiST}

Each MABSC isolate was tested in MGIT 960/TB eXiST system against the four antibiotics indicated. Each drug was tested at multiple concentrations chosen to span the breakpoint concentration recommended by CLSI for BMD method (Table 1$)^{6}$. The growth curves produced automatically by the system were interpreted according to the manufacturer's directions (see Section 3.5 for details).

\subsection{Data analysis}

Categorical results (i.e., susceptible, intermediate, or resistant) obtained using the two methods were compared and classified as follows: concordant, the same categorical result was obtained by both methods; minor error, an intermediate result by one method with a susceptible or resistant result by the other; major error, MGIT 960 incorrectly reported a susceptible isolate as resistant; or very major, MGIT incorrectly reported a resistant isolate as susceptible ${ }^{24}$.

\section{Results}

\subsection{Bacterial strains identification}

Among the 31 MABSC isolates in the study set, all 11 isolates designated $M$. abscessus type 1 by PRAhsp65 typing were identified as M. abscessus subsp. abscessus by rpoB sequencing. Of the $20 \mathrm{M}$. abscessus type 2 isolates, eight were identified as $M$. abscessus subsp. massiliense, nine as $M$. abscessus subsp. bolletii, and three as M. abscessus subsp. abscessus.

\subsection{Minimal inhibitory concentration determination with REMA}

By REMA, ATCC $19977^{\top}$ was susceptible to AMK (MIC <4mg/L), intermediate to FOX (64mg/L), resistant to IPM $(>128 \mathrm{mg} / \mathrm{L})$, and demonstrated inducible resistance to CLR $(<0.5 \mathrm{mg} / \mathrm{L}$ at day 3 and $>64 \mathrm{mg} / \mathrm{L}$ at day 14), Among the 31 clinical isolates the distribution of susceptibility results by microplate testing differed across the antibiotics evaluated and the species of the isolates (Table 2). Clarithromycin demonstrated the most diverse results, with $45 \%$ (14/31) of isolates being susceptible, including $100 \%$ (8/8) of $M$. abscessus subsp. massiliense and none (0/9) of $M$. abscessus subsp. bolletii; $45 \%(14 / 31)$ demonstrated induced resistance and 10\% (3/31) were resistant. In contrast, $97 \%(30 / 31)$ of isolates were susceptible to amikacin, $6.4 \%(2 / 31)$ to cefoxitin, and none to imipenem. 


\subsection{Association of erm(41) and $r /$ genotypes and clarithromycin susceptibility phenotypes}

For all 31 isolates, the genotypes at erm(41) and $r r /$ were compared with the results of CLR microplate susceptibility testing using the Day 14 reading and the subspecies identification (Table 3 ).

PCR analysis indicated the presence of deletions in erm(41) among 11 of the 14 clarithromycinsusceptible isolates, including all $8 \mathrm{M}$. abscessus subsp. massiliense. Of the three susceptible isolates without deletions, all were $M$. abscessus subsp. abscessus that carried the erm(41) T28C point polymorphism ${ }^{25}$. Among the 17 resistant isolates, PCR indicated that all had an intact WT erm(41). Sequencing of $r r$ identified only a single isolate with the A2058G mutation; that isolate also had intact erm(41) and was resistant to clarithromycin. Thus, across all 31 MABSC isolates CLR susceptibility by microplate testing was consistent with erm(41) and rrl genotypes.

\subsection{Inoculum preparation for MGIT 960/TB eXiST susceptibility testing}

Initially, growth of ATCC $19977^{\top}$ was assessed with OADC supplement using dilutions of $10^{-1}, 10^{-2}$, $2 \times 10^{-4}, 10^{-4}, 2 \times 10^{-5}$ and $10^{-8}$. The BACTEC MGIT 960 system requires that the proportional growth control to reach 400 growth units (GU) after 3.0 days. Growth of ATCC $19977^{\top}$ in MGIT tube with OADC reached that threshold after one day of incubation for all dilutions up to $10^{-4}$, and after two and three days of incubation for the $2 \times 10^{-5}$ and $10^{-8}$ dilutions, respectively. Subsequently, the $10^{-4}$ dilution was evaluated with and without OADC enrichment and bacterial growth was detected after two and four days of incubation, respectively. Similar results were obtained with a subset of the clinical isolates. Thus, for MABSC isolates, which have a faster intrinsic growth rate than MTB or slow-growing nontuberculous mycobacteria (NTM), OADC enrichment resulted in accelerated growth rates inconsistent with the timeframes and endpoints specified for the growth control (GC) in the BACTEC MGIT 960 system. Consequently, in all subsequent work OADC was omitted from both growth control and drug testing tubes in MGIT 960, as was already the case in the BMD method.

The appropriate dilution for tubes with drugs was determined using three isolates with different susceptibility profiles for CLR by REMA. The three isolates - ATCC $19977^{\top}$, which demonstrates inducible resistance, plus two clinical isolates, one fully susceptible (isolate 2566) and one constitutively resistant (isolate 1656) - were evaluated using $10^{-2}$ and $10^{-3}$ dilutions prepared as described. Only the $10^{-2}$ dilution consistently provided the expected susceptibility profiles for all three CLR phenotypes (data not shown) and was subsequently confirmed as satisfactory for the other antibiotics.

\subsection{Demonstration of the BACTEC MGIT 960/TB eXiST susceptibility test}

The TB eXiST software monitors each tube for the growth of the organisms over time. For those tubes designated as representing a specific isolate with different concentrations of a single antibiotic plus the growth control, the results are automatically plotted together on one graph whose vertical axis is growth units and horizontal axis, the number of days of incubation. Figure 1 displays the results for an assay of 
ATCC $19977^{\top}$ with CLR. The solid blue line is the growth control (the $10^{-4}$ dilution), which reached $400 \mathrm{GU}$ at 3.5 days. The dotted and dashed blue lines represent $10^{-3}$ and $10^{-2}$ dilutions, respectively, which reached $400 \mathrm{GU}$ in $<72$ hours and, as noted above, would not be valid controls. The other colored lines represent the growth curves in the presence of different concentrations of CLR. The black vertical line (a mix dots and dashes) to the far right marks the endpoint of the assay, which is prespecified in the TB eXiST software as seven days after the GC tube reaches $400 \mathrm{GU}$; in the example shown, $\mathrm{GC}$ reached 400 $\mathrm{GU}$ at 3.5 days, and so the assay endpoint is 10.5 days.

The interpretation of the BACTEC MGIT 960 system is based on the incubation time at which growth at the breakpoint (also referred to as the critical concentration) for the antibiotic being tested (e.g., $2 \mathrm{mg} / \mathrm{L}$ for $\mathrm{CLA}$ ) reaches $100 \mathrm{GU}$ relative to the time the $\mathrm{GC}$ reaches $400 \mathrm{GU}$ and the time of the assay endpoint. Specifically, the isolate is considered resistant if growth in the presence of antibiotic achieves $100 \mathrm{GU}$ before the GC reaches $400 \mathrm{GU}$; intermediate, if it reaches $100 \mathrm{GU}$ after the GC gets to $400 \mathrm{GU}$, but before assay endpoint; and susceptible, if it fails to grow at all or reaches $100 \mathrm{GU}$ only after the assay endpoint. In the assay shown in Figure 1, ATCC $19977^{\top}$ in the presence of CLR $2 \mathrm{mg} / \mathrm{L}$ (green line) achieved $100 \mathrm{GU}$ at 5.6 days incubation, and the isolate is therefore assessed as intermediate susceptibility for CLR. Of note, the organism met criteria for intermediate susceptibility in the presence of CLR across all the concentrations tested ( 0.5 through $8 \mathrm{mg} / \mathrm{L}$ ), consistent with the inducible resistance phenotype demonstrated in REMA.

The results of CLR susceptibility testing for M. abscessus subsp. abscessus isolates 1656 and 2566 using the BACTEC MGIT 960 system are shown in Figures 2 and 3, respectively. At the breakpoint, isolate 1656 achieved $100 \mathrm{GU}$ at 5.25 days, at least 1 full day before the GC curve reached $400 \mathrm{GU}$, and thus meets criteria for resistant. Isolate 2566 demonstrates susceptibility to CLR, with no growth observed in the presence of any drug concentration tested. In media alone isolate 2566 achieved $400 \mathrm{GU}$ at 4.8 days indicating a valid assay. For both isolates, the MGIT 960 results were concordant with MIC testing.

Using the BACTEC MGIT 960 system, ATCC $19977^{\top}$ was assessed as susceptible to AMK, intermediate resistant to FOX, and resistant to IPM (curves not shown). All three results were concordant with MIC testing.

\subsection{Application of BACTEC MGIT 960/TB eXiST susceptibility test}

Using the protocol developed as detailed above, the 31 study isolates were tested for susceptibility to CLR, AMK, FOX, and IPM by BACTEC MGIT 960/TB eXiST system (Table 4) and the results compared to those obtained with MIC susceptibility testing using the REMA protocol (Table 5).

For amikacin, there was $100 \%$ concordance with 30 isolates susceptible and one resistant in both systems. For imipenem, 26 isolates were resistant by both systems. The remaining five isolates were also resistant by MIC testing, but were intermediate by MGIT 960, and thus represented minor errors. 
For cefoxitin, both methods gave the same results for 18 isolates, which included one susceptible, two resistant, and 15 intermediate. All the discrepancies were minor errors, including 12 isolates assessed as intermediate by REMA, but resistant by BACTEC MGIT 960 system and one isolate that was susceptible by REMA, but intermediate by MGIT.

Comparison of the two methods for CLR requires consideration of the phenomenon of inducible resistance, which, using the REMA method, was identified as no growth at 3 days, but growth at some later timepoint up to 14 days. By that technique, all 14 (45.2\%) isolates assessed as susceptible (that is, no growth thru Day 14), were also susceptible by MGIT 960. Microplate identified $3(9.6 \%)$ isolates as resistant (i.e., growth at Day 3), with 14 (45.2\%) demonstrating inducible resistance (i.e., no growth at Day 3 , with growth when monitored at Day 5, 7, 10, or 14). Among those 17 isolates, the MGIT 960 protocol, without modifications, reported $9(29.0 \%)$ as resistant and $8(25.8 \%)$ as intermediate. The specific observations in both methods for the 14 inducible resistant isolates are detailed in Table 6 . There was no apparent correlation between the duration of incubation required to detect inducible resistance by REMA and whether the isolate was reported as intermediate or resistant by BACTEC MGIT 960. Accepting the results for intermediate or resistant by MGIT as equivalent to resistant or inducible-resistant by BMD, then the overall concordance rate for CLR was $100 \%$ (31/31).

\section{Discussion}

Drug susceptibility test of MABSC and other RGM has become of greater clinical importance with the increasing frequency, diversity, and morbidity of infections due to these organisms. The MIC procedures recommended by CLSI are widely applied, but technically demanding. An additional complication, particularly relevant to rapidly-growing mycobacteria, is the recognition of inducible resistance to CLR, which is one of the few agents for which susceptibility by in vitro testing correlates with improved clinical outcomes. Consequently, the accurate detection of inducible resistance is critical, but using a BMD method requires the labor-intensive process of monitoring the culture plates five times over 14 days rather than just once at 3 days. In core laboratories providing DST of NTM to a network of clinical sites, a reliable, automated system would be highly desirable.

The BACTEC MGIT 960 system is an automated system originally applied to drug susceptibility testing of MTB, is recommended by the WHO for that purpose, and is currently in wide use. Recently, the system has been enhanced by release of TB eXiST software that supports the use of multiple protocols, including those developed by end users. Lucke et al. (2012) ${ }^{18}$ applied the MGIT 960/TB eXiST to slowly growing NTM.

This report details development of a protocol for applying the MGIT 960 system to the MABSC, a member of the rapidly growing mycobacteria which have substantially different growth characteristics, and then analyzed the DST results obtained by both the REMA method and the MGIT 960 system for 31 clinical MABSC isolates and four clinically relevant antibiotics (CLR, FOX, IPM, and AMK). The categorical interpretations (i.e., susceptible, intermediate, resistant) were compared at the breakpoints specified by 
CLSI, using the MIC results as the current standard. Across the 124 pairs of results there were no instances of major or very major errors, i.e. no MGIT assays reporting susceptible or resistant where the MIC method reported the opposite. Across all four agents, the MGIT 960 results were strictly concordant in $44(98 \%)$ of 45 instances where MIC testing indicated susceptibility to the antibiotic. The sole exception was a cefoxitin-sensitive isolate reported as intermediate by MGIT 960. Among all the remaining comparisons both systems reported either resistant or intermediate (or in the case of CLR, inducible-resistant). Thus, overall, the MGIT 960 system correctly provided the clinically relevant information - antibiotic susceptible or non-susceptible - for 123 (99.2\%) of the 124 combinations of agents and organisms tested, with the sole exception being in the direction of a conservative discrepancy.

The CLR results were examined in detail to assess the detection of inducible resistance (IR). In conventional 3-day BMD testing, all isolates with IR would be falsely reported as susceptible. Preparing separate CLR microtiter plates and monitoring them over an extended 14-day incubation, although time consuming, proved reliable. Genotyping confirmed that all isolates assessed as either R or IR had intact erm(41) and, conversely, in all isolates assessed as $\mathrm{S}$ that locus was either mutated or absent. This is consistent with studies indicating that the presence of clarithromycin promotes the activation of the erm(41) gene, leading to methylation of the drug binding site and rendering the drug ineffective $\mathrm{e}^{21,23}$.

Of note, no analogous protocol modification was needed for the MGIT 960 system. All isolates that were R or IR by the REMA method were reported as R or I by the MGIT 960 system, and all S isolates gave concordant results with both systems. Thus, applying the standardized protocol described for the MGIT 960 system provided the clinically relevant result, provided that isolates reported as I for CLR by the MGIT 960 were interpreted as resistant.

Both BMD and MGIT 960 methods provide quantitative results, specifically, MIC and time to growth target at critical concentration, respectively. However, an algorithm to compare those very different metrics would be difficult both to develop and interpret. Comparison of the clearly defined categorical interpretations has the advantage of being consistent with established and widely accepted practice. Of particular importance is the principle that susceptibility results based on in vitro growth inhibition cannot be assumed to predict the clinical outcome of infections treated with the drug. Indeed, in the case of agents, such as clarithromycin, that have effects on host inflammatory responses and other factors, it may be clinically appropriate to include the agent in a treatment regimen even when in vitro testing does not indicate susceptibility ${ }^{26}$.

The primary limitations to our study relate to its restricted scale, including number of isolates, number of antibiotics, diversity of susceptibility phenotypes for some combinations of antibiotics and subspecies, limited genetic analyses, and involvement of a single laboratory. To address these issues and formally validate the protocol will require a substantially larger study.

Both BMD and BACTEC MGIT 960 methods have strengths and limitations. BMD requires modest investments in equipment (e.g., incubators) and supplies (microplates). However, preparing microplates 
with multiple different antibiotics involves dispensing multiple drug stocks solutions and performing different serial dilutions on a single plate, which increases the risk of pipetting errors. As noted above, visual reading of the plates is entirely manual and requires extensive training; testing for CLR inducible resistance, as needed for MABSC, involves appreciable added effort. The BACTEC MGIT 960 system is technically easier to perform, demands less manipulation, provides an automated reading, and, applying the protocol presented here, provides reliable detection of CLR inducible resistance without modification. Further, the use of screw capped culture tubes together with the automated reading provides a higher biosafety level and a lower risk of test contamination. The greater capital and maintenance costs of the MGIT 960 make it more appropriate for larger, often centralized, laboratories with higher workloads. One potential benefit in demonstrating the system can be adapted to additional classes of mycobacteria may be to make it more applicable for additional sites.

\section{Conclusion}

This report describes the development and initial assessment of a protocol for DST of MABSC using the BACTEC MGIT 960/TB eXiST system, already validated for M. tuberculosis ${ }^{7}$ and previously demonstrated applicable to slow-growing mycobacteria. ${ }^{18}$ These observations are consistent with expectations and further suggest that the system can be applied to other rapidly-growing mycobacteria. Thus, for laboratories that have, or are considering acquiring, the MGIT 960, the results of this study suggest that a single DST methodology, with multiple advantages as noted above, can be applied broadly across nontuberculous mycobacteria as well as $M$. tuberculosis. We look forward to participating in the full-scale study, representing a collaboration of multiple laboratories and disciplines, that is required to fully validate our results and support that potential.

\section{Abbreviations}

MABSC - Mycobacterium abscessus complex

RGM - Rapidly growing mycobacteria

CF - Cystic fibrosis

DST - Drug susceptibility testing

CLSI - Clinical and Laboratory Standards Institute

MIC - Minimal inhibitory concentration

CLR - Clarithromycin

MGIT 960 - Mycobacterium Growth Indicator Tube 960

WHO - World Health Organization 
MTB - Mycobacterium tuberculosis

REMA - resazurin staining

AMK - Amikacin

FOX - Cefoxitin

IPM - Imipenem

ATS - American Thoracic Society

CAMHB - Cation-adjusted Mueller-Hinton broth

OADC - Oleic Albumin Dextrose Catalase

IR - Inducible resistance

GU - Growth units

GC - Growth control

NTM - Nontuberculous mycobacteria

\section{Declarations}

\section{Acknowledgements}

We thank Dr. Miguel Viveiros and Diana Machado, from Universidade Nova de Lisboa, for critical reading of the manuscript.

\section{Funding}

This work was supported by Fundação de Amparo à Pesquisa do Estado de São Paulo (FAPESP) (grant number 2014/50094-4). Technical support was offered by BD for the use of MGIT960/EpiCenter TB eXisT.

\section{Transparency declarations}

None to declare.

\section{References}

1. Medjahed H, Gaillard JL, Reyrat JM. Mycobacterium abscessus: a new player in the mycobacterial field. Trends Microbiol 2010;18:117-23.

2. Petrini B. Mycobacterium abscessus: an emerging rapid-growing potential pathogen. APMIS 2006;114:319-28. 


\section{Lee MR, Sheng WH, Hung CC et al. Mycobacterium abscessuscomplex infections in humans. Emerg Infect Dis 2015; 21:1638-1646.}

4. Griffith DE, Brown-Elliott BA, Benwill JL. Mycobacterium abscessus "Pleased to Meet you, Hope You Guess My Name." Ann Am ThoracSoc 2015;3:436-439.

5. Stout JE, Koh WJ, Yew WW. Update on pulmonary disease due to non-tuberculous mycobacteria. Int $\mathrm{J}$ Infect Dis 2016;45:123-34.

6. Clinical and Laboratory Standards Institute (CLSI). Susceptibility Testing of Mycobacteria, Nocardiae, and Other Aerobic Actinomycetes; Approved Standard-Third Edition. CLSI M62 (ISBN 978-1-68440027-0). Clinical and Laboratory Standards Institute, 940 West Valley Road, Suite 1400, Wayne, Pennsylvania 19087-1898 USA, 2018.

7. Palomino JC, Martin A, Camacho M, Ghuerra H, Swings J, Portaels F. Resazurin microtiter assay plate: simple and inexpensive method for detection of drug resistance in Mycobacterium tuberculosis. Antimicrob Agents Chemother 2002;46:2720-2.

8. Martin A, Camacho M, Portaels F, Palomino JC. Resazurin microtiter assay plate testing of Mycobacterium tuberculosis susceptibilities to second-line drugs: rapid, simple and inexpensive method. Antimicrob Agents Chemother 2003;47:3616-19.

9. Montoro E, Lemus D, Echemendia M, Martin A, Portaels F, Palomino JC 2005. Comparative evaluation of the nitrate reduction assay, the MTT test, and the resazurin microtiter assay for drug susceptibility testing of clinical isolates of Mycobacterium tuberculosis. J Antimicrob Chemother 2005;55: 500-5.

10. Carvalho NFG, Sato DN, Pavan FR et al. Resazurin Microtiter Assay for Clarithromycin Susceptibility Testing of Clinical Isolates of Mycobacterium abscessus J Clin Lab Anal 2016;30:751-5.

11. RamisIB, Cnockaert M, von Groll A, Nogueira CL, Leão SC, Andre E, Simon A, Palomino JC, da Silva $P E$, Vandamme $P$, Martin A 2015. Antimicrobial susceptibility of rapidly growing mycobacteria using the rapid colorimetric method. Eur J Clin Microbiol Infect Dis 2015; 34(7):1403-13.

12. Ramis IB, Vianna JS, Silva Junior L, von Groll A, Ramos DF, Lobo MM, Zanatta N, Viveiros M, Silva PEAD.Tuberculosis (Edinb). 2019 Sep;118:101853.

13. Martin A, Bouyakoub Y, Soumillion K, Mantu EON et al. Targeting bedaquiline mycobacterial efflux pump to potentially enhance therapy in Mycobacteriumabscessus. Int J Mycobacteriol. 2020;9(1):7175 .

14. Use of liquid TB culture and drug susceptibility testing (DST) in low media and medium income settings. Geneva, World Health Organization, 2008. Available at: http://www.stoptb.org/wg/gli/assets/ documents/EGM\%20report_Use\%20of\%20Liquid\%20Culture\%20Media.pdf.

15. Policy guidance on drug-susceptibility testing (DST) of second-line antituberculosis drugs. Geneva, World Health Organization, 2008 (WHO/HTM/TB/2008.392). Available at: http://whqlibdoc.who.int/hq/ 20008/WHO_HTM_TB_2008.392_eng.pdf?ua=1. 
16. Giampaglia CM, Martins MC, Vieira GB, et al. Multicentre evaluation of an automated BACTEC 960 system for susceptibility testing of Mycobacterium tuberculosis. Int J Tuberc Lung Dis 2007;11:98691.

17. Hombach M, Somoskövi A, Hömke R et al. Drug susceptibility distributions in slowly growing nontuberculous mycobacteria using MGIT 960 TB eXiST. Int J Med Microbiol 2013;303:270-6.

18. Lucke K, Hombach M, Friedel U et al. Automated quantitative drug susceptibility testing of nontuberculous mycobacteria using MGIT 960/EpiCenter TB eXiST. J Antimicrob Chemother 2012;67:154-8.

19. Adékambi T, Colson P, Drancourt M. rpoB-based identification of nonpigmented and late-pigmenting rapidly growing mycobacteria. J Clin Microbiol 2003;41:5699-708.

20. Chimara E, Ferrazoli L, Ueky SY et al. Reliable identification of mycobacterial species by PCR restriction enzyme analysis (PRA)-hsp65 in a reference laboratory and elaboration of a sequencebased extended algorithm of PRA-hsp65 BMC Microbiology 2008;8:48.

21. Carvalho NFG, Pavan F, Sato DN et al. Genetic correlates of clarithromycin susceptibility among isolates of the Mycobacterium abscessus group and the potential clinical applicability of a PCRbased analysis of erm(41). J Antimicrob Chemother 2018;73:862-866.

22. Griffith DE, Aksamit T, Brown-Elliott BA et al. An official ATS/IDSA statement: diagnosis, treatment, and prevention of nontuberculous mycobacterial diseases. Am J Respir Crit Care Med 2007;175:367416.

23. Maurer FP, Rüegger V, Ritter $\mathrm{C}$ et al. Acquisition of clarithromycin resistance mutations in the $23 \mathrm{~S}$ rRNA gene of Mycobacterium abscessus in the presence of inducible erm(41). J Antimicrob Chemother 2012;67:2606-11.

24. Landis JR, Koch GG. The measurement of observer agreement for categorical data. Biometrics 1977;33:159-174.

25. Brown-Elliott BA, Vasireddy S, Vasireddy R et al. Utility of sequencing the erm(41) gene in isolates of Mycobacterium abscessus abscessus with low and intermediate clarithromycin MICs. J Clin Microbiol. 2015;53(4):1211-5.

26. Delay, CL, laccarino JM, Lange $M$ et al. Treatment of nontuberculous mycobacterial pulmonary disease: an official ATS/ERS/ESCMID/IDSA clinical practice guideline. Eur Respir J 2020 Jul 7;56(1):2000535.

\section{Tables}

Table 1. Drugs concentrations used for susceptibility testing in the BACTEC MGIT960 system and the breakpoints of each drug. 


\begin{tabular}{|l|c|c|c|c|c|c|c|c|c|}
\hline Drugs & \multicolumn{7}{|c|}{ Concentrations used in the MGIT960 system (mg/L) } \\
\hline & 0.5 & 1 & 2 & 4 & 8 & 16 & 32 & 64 & 128 \\
\hline Clarithromycin (CLR) & $\mathrm{X}$ & $\mathrm{X}$ & $\mathrm{BP}$ & $\mathrm{I}$ & $\mathrm{R}$ & & & & \\
\hline Imipenem (IPM) & & & & $\mathrm{BP}$ & $\mathrm{I}$ & $\mathrm{I}$ & $\mathrm{R}$ & & \\
\hline Amikacin (AMK) & & & & & & $\mathrm{BP}$ & $\mathrm{I}$ & $\mathrm{R}$ & \\
\hline Cefoxitin (FOX) & & & & & & $\mathrm{BP}$ & $\mathrm{I}$ & $\mathrm{I}$ & $\mathrm{R}$ \\
\hline
\end{tabular}

BP, breakpoint for susceptibility, the MIC that defines a susceptible isolate (CLSI, 2018).

I, intermediate; R, resistant; $\mathrm{X}$, additional concentrations tested;

Table 2. Drug susceptibility profiles by microplate method for MABSC isolates by species.

\begin{tabular}{|c|c|c|c|c|c|c|c|c|c|c|c|c|}
\hline \multirow[t]{2}{*}{ Species } & \multicolumn{3}{|c|}{ CLR } & \multicolumn{3}{|c|}{ FOX } & \multicolumn{3}{|c|}{ IPM } & \multicolumn{3}{|c|}{ AMK } \\
\hline & $\begin{array}{c}S \\
(N=14)\end{array}$ & $\begin{array}{c}\text { IR } \\
(\mathrm{N}=14)\end{array}$ & $\begin{array}{c}R \\
(N=3)\end{array}$ & $\begin{array}{c}S \\
(N=2)\end{array}$ & $\begin{array}{c}\mathrm{I} \\
(\mathrm{N}=27)\end{array}$ & $\begin{array}{c}R \\
(N=2)\end{array}$ & $\begin{array}{c}\mathrm{S} \\
(\mathrm{N}=0)\end{array}$ & $\begin{array}{c}\mathrm{I} \\
(\mathrm{N}=0)\end{array}$ & $\begin{array}{c}R \\
(N=31)\end{array}$ & $\begin{array}{c}S \\
(N=30)\end{array}$ & $\begin{array}{c}\mathrm{I} \\
(\mathrm{N}=0)\end{array}$ & $\begin{array}{c}\mathrm{R} \\
(\mathrm{N}=1)\end{array}$ \\
\hline $\begin{array}{l}\text { M. } \\
\text { abscessus }\end{array}$ & 6 & 6 & 2 & 1 & 12 & 1 & - & - & 14 & 13 & - & 1 \\
\hline M. bolletii & - & 8 & 1 & - & 8 & 1 & - & - & 9 & 9 & - & - \\
\hline $\begin{array}{l}\text { M. } \\
\text { massiliense }\end{array}$ & 8 & - & - & 1 & 7 & - & - & - & 8 & 8 & - & - \\
\hline
\end{tabular}

S: susceptible, I: intermediate, IR: induced resistant, R: resistant. CLR: clarithromycin; FOX: cefoxitin; IPM: imipenem; AMK: amikacin.

Table 3. Clarithromycin susceptibility by microplate method after 14 days incubation and genotype profile of the erm(41) and rrl genes.

\begin{tabular}{|c|c|c|c|c|c|c|c|c|}
\hline \multicolumn{2}{|c|}{ Genotype } & \multicolumn{4}{|c|}{ Susceptibility to $C_{L R}{ }^{a}$} & \multicolumn{3}{|c|}{ Subspecies within $M$. abscessus complex } \\
\hline$r r l$ & $\operatorname{erm}(41)$ & $\mathbf{N}$ & $\underset{(N=14)}{S}$ & $\underset{(\mathrm{N}=14)}{\mathrm{IR}}$ & $\underset{(\mathrm{N}=3)}{\mathrm{R}}$ & $\begin{array}{c}\text { M. abscessus } \\
(\mathrm{N}=14)\end{array}$ & $\begin{array}{c}\text { M. bolletii } \\
(\mathrm{N}=9)\end{array}$ & M. $\underset{(\mathrm{N}=8)}{\operatorname{massiliense}}$ \\
\hline WT & WT & 16 & - & 14 & 2 & 7 & 9 & - \\
\hline A2058G & WT & 1 & - & - & 1 & 1 & - & - \\
\hline WT & Deletions $^{b}$ & 11 & 11 & - & - & 3 & - & 8 \\
\hline WT & $\mathrm{T} 28 \mathrm{C}$ & 3 & 3 & - & - & 3 & - & - \\
\hline A2058G & $\mathrm{T} 28 \mathrm{C}$ & - & - & - & - & - & - & - \\
\hline
\end{tabular}

\footnotetext{
a. CLR, clarithromycin; S, susceptible after 14-days incubation; R, resistant after 3-days incubation; IR: inducible resistance demonstrated during extended 14-day incubation. See text for details.

b. Two deletions in erm(41) of 2 bp (nucleotides 64-65) and 274 bp (nucleotides 159-432)
}

Table 4. Drug susceptibility results obtained by the BACTEC MGIT 960/EpiCenter TB eXiST method for MABSC isolates by species.

\begin{tabular}{lccc|ccc|ccc|ccc}
\hline Subspecies & \multicolumn{3}{c}{ CLR } & \multicolumn{3}{c|}{ FOX } & \multicolumn{3}{c|}{ IPM } & \multicolumn{3}{c}{ AMK } \\
& S & I & R & S & I & R & S & I & R & S & I & R \\
\cline { 2 - 11 } M. abscessus & 6 & 5 & 3 & 1 & 9 & 4 & - & 2 & 12 & 13 & - & 1 \\
M. bolletii & - & 2 & 7 & - & 4 & 5 & - & 3 & 6 & 9 & - & - \\
\hline M. massiliense & 8 & - & - & - & 3 & 5 & - & - & 8 & 8 & - & - \\
\hline
\end{tabular}


S: susceptible, I: intermediate, R: resistant.

Table 5. Comparison of the drugs susceptibility profiles of 31 MAG isolates for amikacin, imipenem, cefoxitin, and clarithromycin obtained by the microplate MIC and MGIT 960 methods.

\begin{tabular}{ccc|c|c|c|}
\hline Antibiotic & Crit. Conc. & \multicolumn{1}{c}{ MGT 960 } & \multicolumn{3}{c}{ REMA } \\
\hline AMK & $16 \mathrm{mg} / \mathrm{L}$ & & $\mathrm{S}$ & $\mathrm{I}$ & $\mathrm{R}$ \\
\cline { 3 - 5 } & & $\mathrm{S}$ & 30 & - & - \\
\hline & & $\mathrm{I}$ & - & - & - \\
\hline $\mathrm{R}$ & - & - & 1 \\
\hline
\end{tabular}

\begin{tabular}{|c|c|c|c|c|c|}
\hline \multirow[t]{4}{*}{ IPM } & \multirow[t]{4}{*}{$4 \mathrm{mg} / \mathrm{L}$} & & $\mathrm{S}$ & $\mathrm{I}$ & $\mathrm{R}$ \\
\hline & & $\mathrm{S}$ & - & - & - \\
\hline & & $\mathrm{I}$ & - & - & 5 \\
\hline & & $\mathrm{R}$ & - & - & 26 \\
\hline
\end{tabular}

\begin{tabular}{|c|c|c|c|c|c|}
\hline \multirow[t]{4}{*}{ FOX } & \multirow[t]{4}{*}{$16 \mathrm{mg} / \mathrm{L}$} & & $\mathrm{S}$ & $\mathrm{I}$ & $\mathrm{R}$ \\
\hline & & $\mathrm{S}$ & 1 & - & - \\
\hline & & $\mathrm{I}$ & 1 & 15 & - \\
\hline & & $\mathrm{R}$ & - & 12 & 2 \\
\hline
\end{tabular}

\begin{tabular}{l|c|c|c|c|}
\hline CLR 2 mg/L & & $\mathrm{S}$ & $\mathrm{IR}$ & $\mathrm{R}$ \\
\cline { 2 - 5 } & $\mathrm{S}$ & 14 & - & - \\
\hline $\mathrm{I}$ & - & 7 & 1 \\
\hline $\mathrm{R}$ & - & 7 & 2 \\
\hline & \multicolumn{4}{|c}{} \\
\hline
\end{tabular}

Antibiotics: CLR, clarithromycin; FOX, cefoxitin; IPM, imipenem; AMK, amikacin.

Susceptibility: S, susceptible; IR, inducible resistance (applicable only to CLA by microplate MIC with extended 14-day incubation); I, intermediate; R, resistant.

Table 6. Comparison between the time of detection of resistance to clarithromycin in 17 isolates by microplate MIC compared with results of DST using the MGIT 960 system. 


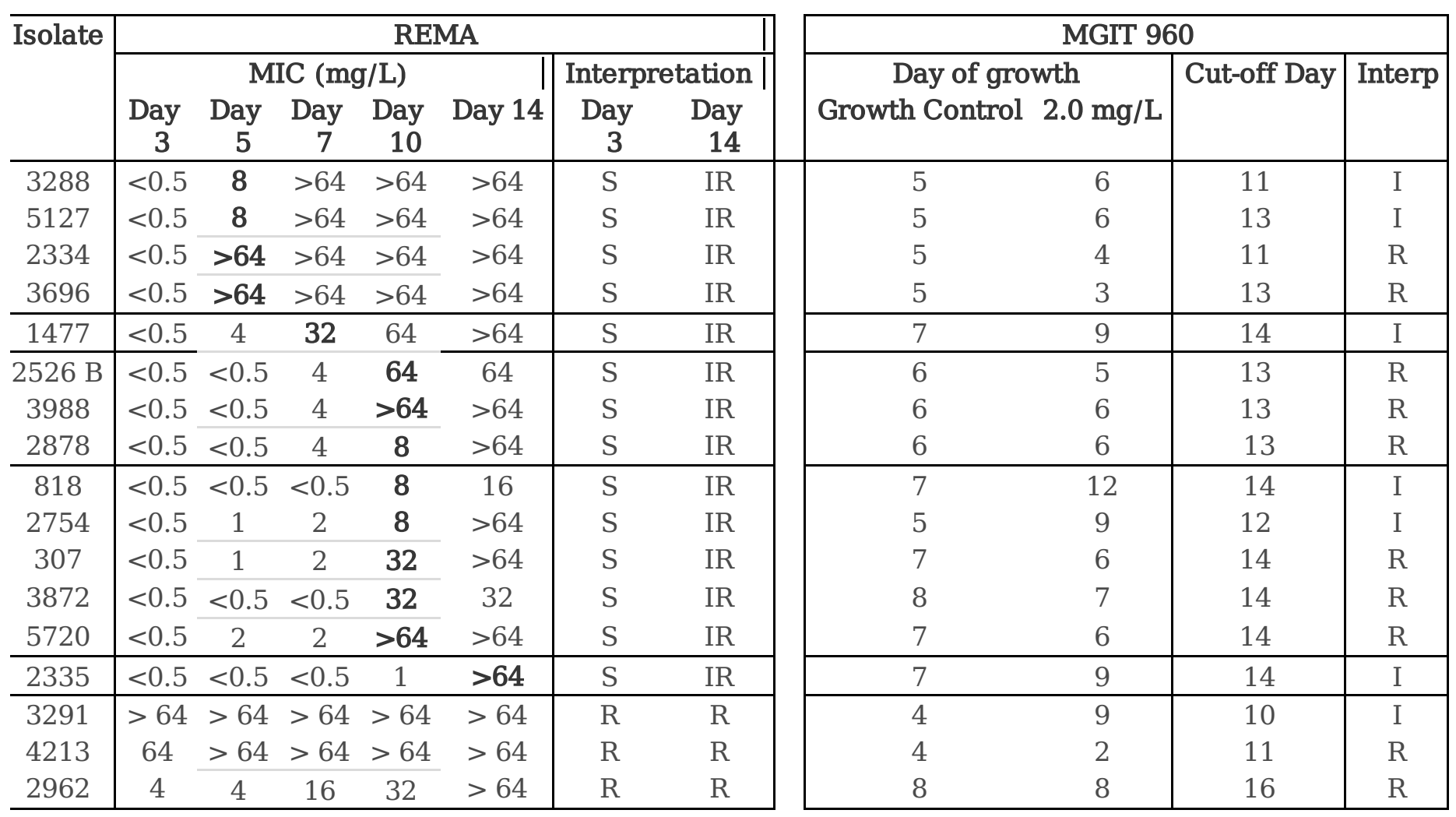

S: susceptible, I: intermediate, IR: induced resistant, R: resistant. For REMA, on the day inducible resistance was detected (MIC $\geq 8 \mathrm{mg} / \mathrm{L}$ ), the observed MIC is shown in bold.

\section{Figures}

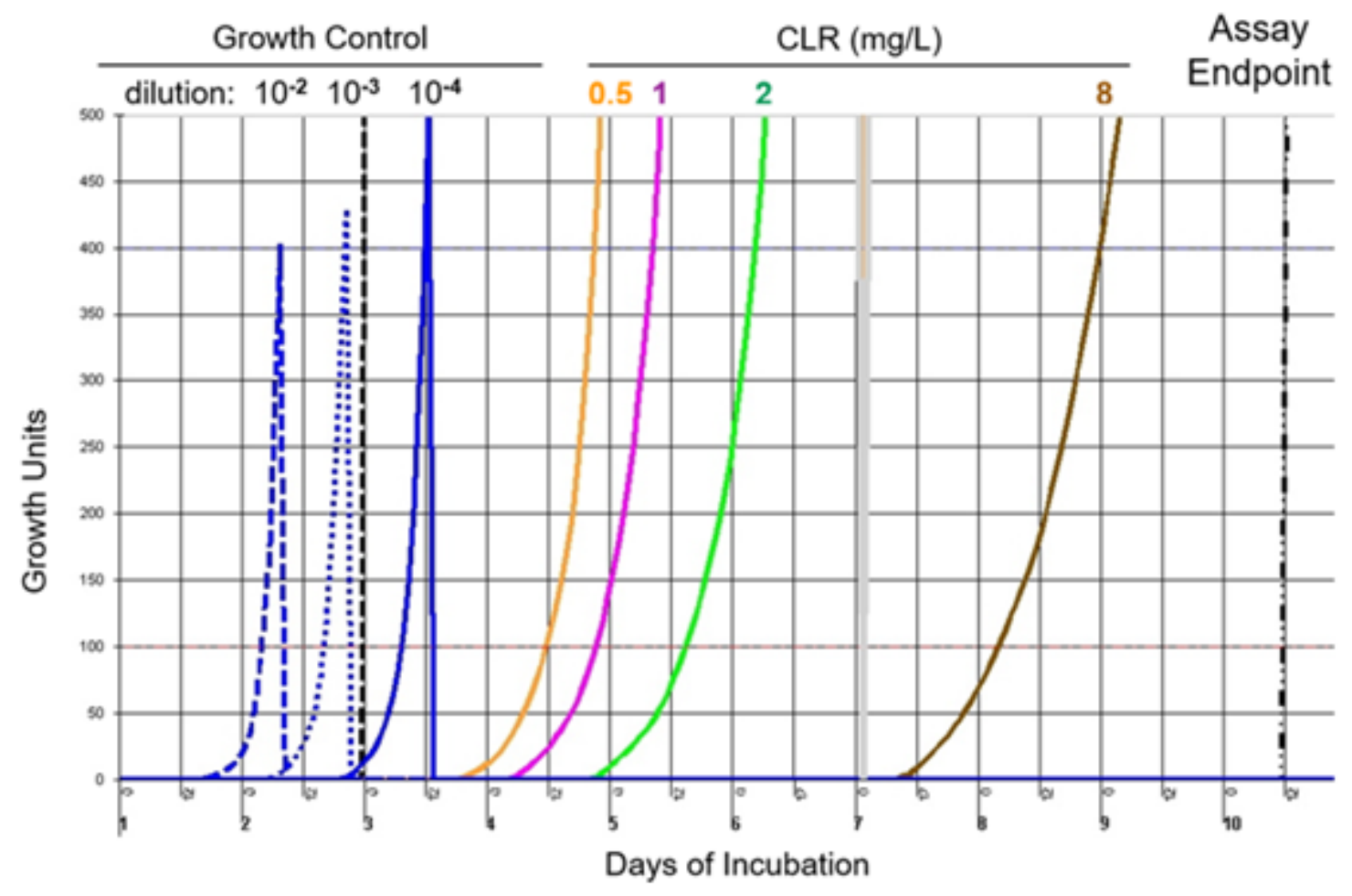

Page $16 / 18$ 


\section{Figure 1}

Growth curves of M. abscessus ATCC 19977T in MGIT 960 system. Growth control tubes (without drug) were inoculated with dilutions of 10-2 (dashed blue), 10-3 (dotted blue) and 10-4 (solid blue). Only the 104 dilution achieved 400 Growth Units (GU) after 3.0 days of incubation. The other dilutions reached that growth level too quickly to be valid controls. All tubes with CLR were inoculated with the 10-2 dilution. At the breakpoint for CLR (2 mg/L), the culture achieved $100 \mathrm{GU}$ at 5.6 days, after the growth control (3.5 days) and before the prespecified assay endpoint (10.5 days, i.e., 7 days after the growth control; dasheddotted vertical black line). Consequently, ATCC 19977T was classified as intermediate (I) susceptibility.

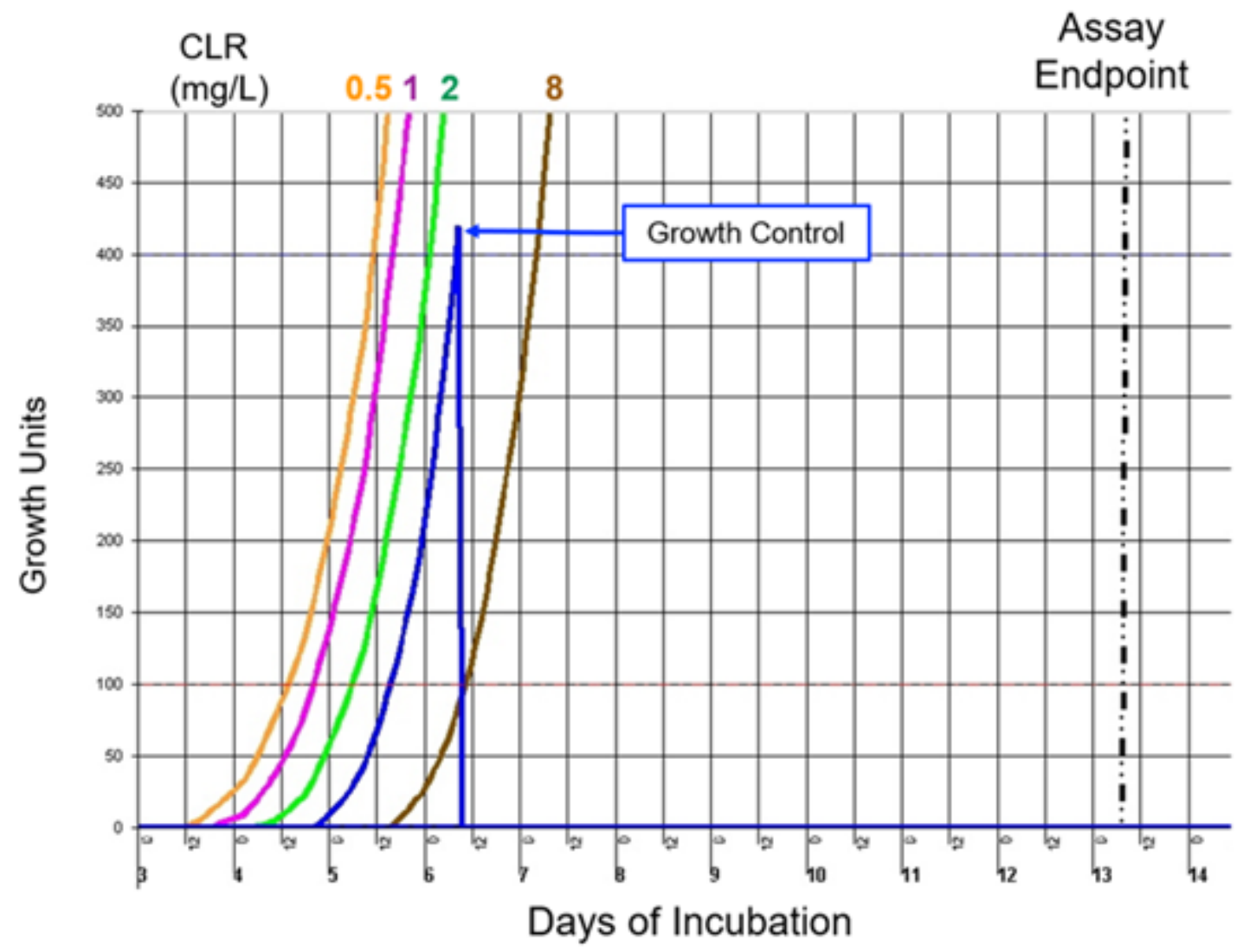

\section{Figure 2}

Growth curves of M. abscessus subsp. abscessus isolate \#1656 in MGIT 960 system, including Growth Control (without drug - in blue) and with CLR at 0.5 (orange), 1 (pink), 2 (green), and 8 mg/L (brown). At the susceptibility breakpoint ( $2 \mathrm{mg} / \mathrm{L}$ ), the organism achieved $100 \mathrm{GU}$ at 5.25 days, at least 1 full day before the Growth Control reached $400 \mathrm{GU}$, and, therefore, the isolate was assessed as resistant (R). 


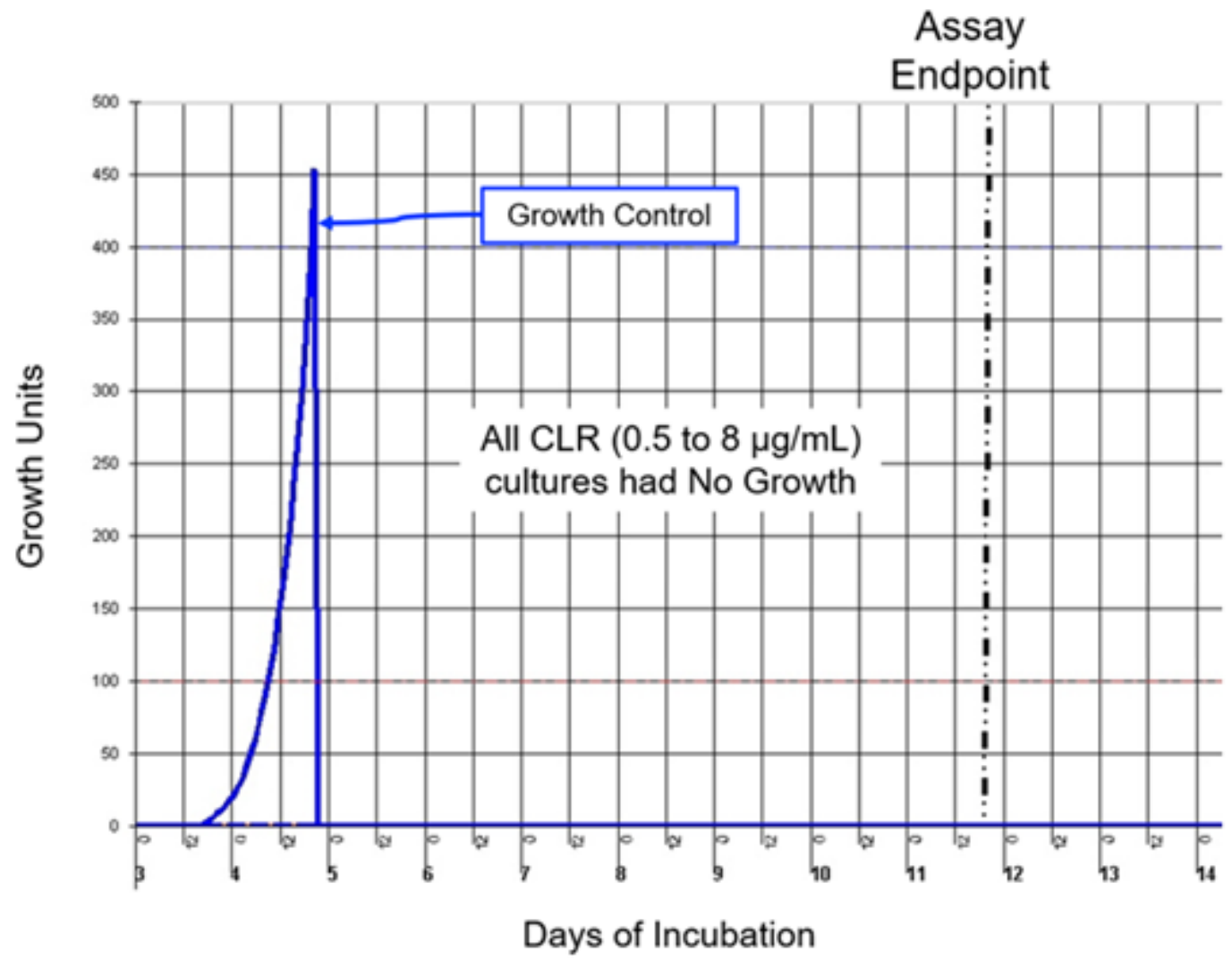

Figure 3

Growth curves of M. abscessus subsp. massiliense isolate \#2566 in MGIT 960 system, including Growth Control (without drug). In parallel, the same inoculum was incubated in cultures with CLR at 0.5, 1, 2, and $8 \mathrm{mg} / \mathrm{L}$ and no growth was detected at any time. Therefore, the isolate was assessed as susceptible (S). 